\title{
Perforación de íleon relacionada con el uso de resinas de intercambio iónico: informe de un caso
}

\author{
Jorge E. Rico-Fontalvo ${ }^{1}$, Nehomar Pájaro-Galvis² ${ }^{2 *}$ Rodrigo Daza-Arnedo³, Emilio Rey-Vela ${ }^{4}$, \\ Emir Ortiz-Ibarra', Víctor Leal-Martínez², Luis G. Salgado-Montie/5 y Juan C. Villa ${ }^{6}$
}

${ }^{1}$ Servicio de Medicina Interna, Clínica SOMA, Medellín, Antioquia, Colombia; ${ }^{2}$ Servicio de Medicina Interna, Facultad de Ciencias de la Salud, Universidad del Sinú, Cartagena, Bolívar; ${ }^{3}$ Servicio de Medicina Interna, Nuevo Hospital Bocagrande, Cartagena, Bolívar; ${ }^{4}$ Unidad de Cuidados Intensivos, Hospital Universitario la Samaritana, Bogotá, Colombia; ${ }^{5}$ Unidad de Cuidados Intensivos, Nuevo Hospital Bocagrande, Cartagena, Bolívar; ${ }^{6}$ Servicio de Anestesiología, Clínica SOMA, Medellín, Antioquia, Colombia

\section{Resumen}

La hiperpotasemia es una de las complicaciones hidroelectrolíticas más frecuentes en los pacientes con lesión renal aguda o enfermedad renal crónica e incrementa el riesgo de arritmias cardiacas letales. Para su tratamiento agudo se utilizan, entre otras medidas, las resinas de intercambio iónico (poliestireno sulfonato sódico, poliestireno sulfonato cálcico), así como otras modalidades disponibles en fecha reciente para la quelación intestinal del potasio, entre ellas patiromer o ciclosilicato de zirconio. El poliestireno sulfonato sódico y el poliestireno sulfonato cálcico se consideran fármacos muy seguros, pero producen reacciones adversas graves, por fortuna raras. Se describe el caso de un paciente con insuficiencia renal crónica en estadio $V$ en hemodiálisis, quien sufre una perforación espontánea del íleon relacionada con el uso de resinas de intercambio iónico, un episodio adverso poco frecuente y con escasos informes en las publicaciones médicas.

Palabras clave: Insuficiencia renal crónica. Hiperpotasemia. Resinas de intercambio iónico. Perforación intestinal.

\section{Ileon perforation associated with the use of ion exchange resins, a case report}

\begin{abstract}
Hyperkalemia is one of the most frequent hydroelectrolytic complications in patients with acute kidney injury or chronic kidney disease, which increases the risk of fatal cardiac arrhythmias. For its acute management, among other measures, ion exchange resins (sodium polystyrene sulfonate; calcium polystyrene sulfonate) are used, as well as other recently available strategies for intestinal chelation of potassium, such as patiromer or zirconium cyclosilicate; being sodium polystyrene sulfonate and calcium polystyrene sulfonate, medicines considered very safe, but which have serious adverse reactions, which fortunately are rare. We present the case of a patient with stage $V$ chronic kidney failure on hemodialysis who presents with spontaneous ileal perforation associated with the use of ion exchange resins; infrequent adverse event and with few reports in the literature.
\end{abstract}

Key words: Chronic kidney failure. Hyperkalemia. lon-exchange resins. Intestinal perforation.

\section{Correspondencia:}

*Nehomar Pájaro-Galvis

E-mail: neho94@ hotmail.com
Disponible en internet: 18-06-2021

Nefro Latinoam. 2021;18:80-83 www.nefrologialatinoamericana.com licencia CC BY-NC-ND (http://creativecommons.org/licenses/by-nc-nd/4.0/). 


\section{Introducción}

Las resinas de intercambio iónico son fármacos utilizados de forma amplia para el tratamiento de la hiperpotasemia de cualquier origen, pero sobre todo en los pacientes con insuficiencia renal crónica. Una vez alcanzada la luz intestinal, su mecanismo de acción consiste en intercambiar los iones calcio o sodio de la resina (según sea el tipo) por el ion potasio, de tal modo que este último aumenta su excreción por las heces. Aunque sus efectos secundarios son raros, pueden ser letales e incluyen necrosis intestinal, ulceraciones, perforación y sangrado. Es fundamental un índice elevado de sospecha para establecer un diagnóstico oportuno debido a que, dada la escasa frecuencia de estas complicaciones, casi nunca se consideran en el diagnóstico diferencial.

\section{Presentación del caso}

Paciente masculino de 64 años de edad con antecedentes de obesidad, neumopatía crónica, hipertensión arterial mal controlada y enfermedad renal crónica en estadio 5 , con programa de hemodiálisis y sin función renal residual; se hallaba bajo tratamiento farmacológico con amitriptilina (25 mg/día VO), ácido fólico ( $1 \mathrm{mg} /$ día VO), metoprolol (50 mg/12 h VO), losartán (50 mg/12 h VO), omeprazol (20 mg/día), prazosina (1 mg/8 h VO) y bisacodilo a necesidad. Ingresó al servicio de urgencias de un hospital de mediana complejidad de la ciudad de Medellín, con un cuadro clínico de 12 días de evolución caracterizado por cefalea biparietal continua exacerbada con las maniobras de Valsalva y dificultad para conciliar el sueño. Sin antecedente de traumatismos. La TC cerebral sin contraste identifica hematoma subdural hemisférico derecho subagudo, con algunas imágenes hiperdensas que sugieren sangrado reciente frontoparietal derecho con efecto de masa secundario. Cuatro días después de su ingreso se lo remite a la institución de los autores (Clínica SOMA) debido a la imposibilidad de instituir tratamiento dialítico. A su ingreso tiene los siguientes estudios de laboratorio: BUN, $57.1 \mathrm{mg} / \mathrm{dl}$; creatinina, $10.41 \mathrm{mg} / \mathrm{dl}$; potasio, $5.1 \mathrm{meq} / \mathrm{L}$; sodio, $138 \mathrm{meq} / \mathrm{L}$; cloro, $103.8 \mathrm{meq} / \mathrm{L} ; \mathrm{Ca}, 8.7 \mathrm{mg} / \mathrm{dl}$; magnesio, $2.16 \mathrm{mg} / \mathrm{dl}$; leucocitos, $8,900 / \mathrm{mm}^{3}$; neutrófilos, $48 \%$; linfocitos,

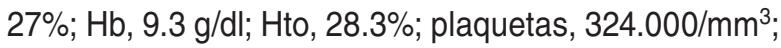
INR, $0.88 \mathrm{~s}$; TPT, $27.63 \mathrm{~s}$; TP, $12.9 \mathrm{~s}$; albúmina, $3.4 \mathrm{~g} / \mathrm{dl}$. Al ingresar a la institución se programa para craneotomía para drenaje, sin complicaciones intraoperatorias. En el sexto día de hospitalización, en virtud de la persistencia de la encefalopatía, se realiza control tomográfico que delinea una nueva complicación con isquemia en el territorio de la arteria cerebral posterior derecha.

Durante la evolución muestra poca regularidad con el tratamiento dialítico y menor duración de las sesiones de hemodiálisis debido a una disfunción del acceso vascular yugular derecho, lo que produce hiperpotasemia (potasio, $6.33 \mathrm{meq} / \mathrm{L}$; BUN, 27mg/dl; creatinina, $5.88 \mathrm{mg} / \mathrm{dl}$ ) tratada por el servicio de cuidados intensivos con medidas farmacológicas, incluidas las resinas de intercambio iónico (poliestireno sulfonato cálcico) a dosis de $45 \mathrm{~g}$ orales al día (de manera inicial entre los días 4 y 5 de hospitalización y luego en los días 22 a 24 de hospitalización en virtud de nuevos valores de potasio sérico de $7.1 \mathrm{meq} / \mathrm{L})$.

El día 25 de hospitalización refiere dolor abdominal de rápido inicio, difuso y con acentuación en fosa iliaca derecha, difícil de valorar por la encefalopatía, pero sin signos de irritación peritoneal. El servicio de cirugía general lo valora y se solicita TAC de abdomen; ésta identifica signos de obstrucción intestinal con la zona de transición a nivel del íleon distal y signo de "remolino" de los vasos mesentéricos que indica posible vólvulo del íleon distal. Se considera incongruente la descripción radiológica con los hallazgos del paciente y su historia clínica, por lo que se indica una laparoscopia diagnóstica que reconoce en el íleon distal, a $\sim 40 \mathrm{~cm}$ de la válvula ileocecal, un plastrón de intestino delgado sin volvulación. Se libera el plastrón y se identifica un absceso alojado contra la pared abdominal, en relación con una perforación del intestino delgado de $\sim 1 \mathrm{~cm}$, con efecto obstructivo y con bordes necróticos y muy acartonados. Se practica una resección de unos $50 \mathrm{~cm}$ de intestino delgado más anastomosis terminoterminal. La muestra se envía a patología, que confirma lesión isquémica grave de la mucosa del íleon, con varios cristales de resina de intercambio iónico en la superficie, un cuadro consistente con perforación intestinal relacionada con el uso de poliestireno sulfonato cálcico (Fig. 1 a 3). Se instituye antibioticoterapia de amplio espectro con meropenem por ocho días. Después del procedimiento quirúrgico se observa una evolución clínica satisfactoria. El día 39 de hospitalización recibe el alta en buenas condiciones y sin déficit neurológico.

\section{Discusión}

Las resinas de intercambio iónico se administran de modo extenso para el tratamiento de la hiperpotasemia, 


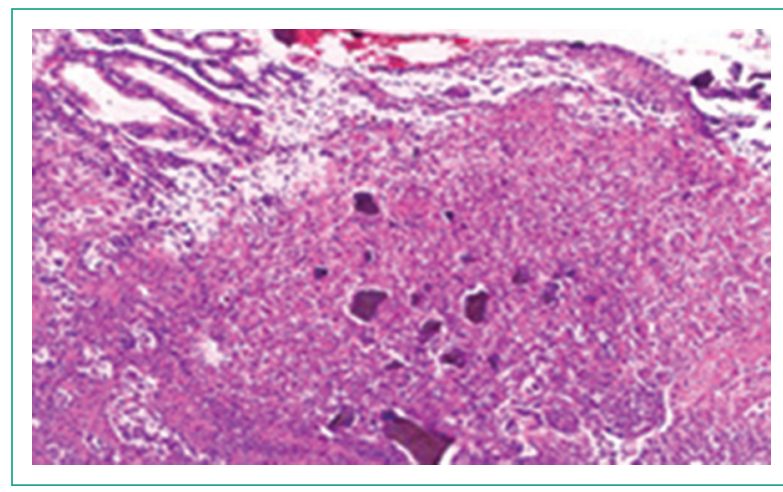

Figura 1. Íleon con lesión isquémica. Se observan cristales de resinas de intercambio iónico.

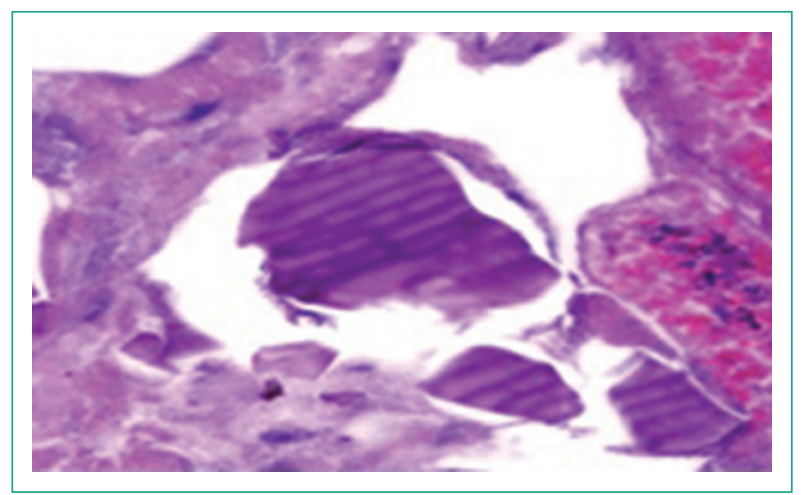

Figura 2. Los cristales muestran una apariencia agrietada y adoptan un color púrpura en la tinción HE.

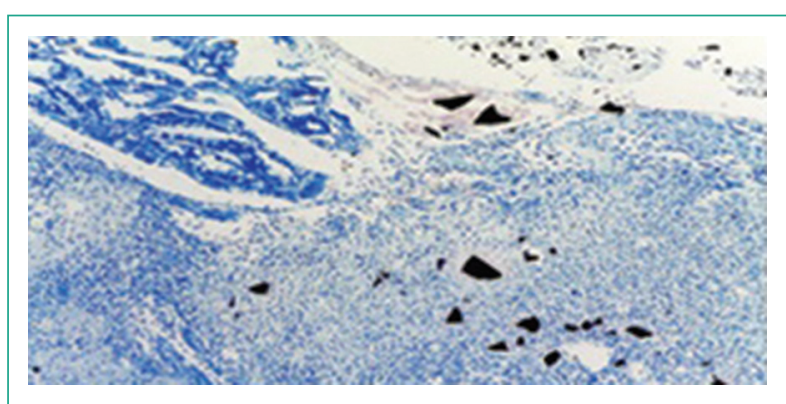

Figura 3. Los cristales absorben casi cualquier coloración de histoquímica. En la tinción ZN asumen un color negro. ZN: tinción de ziehl neelsen.

en particular en pacientes con enfermedad renal crónica, aunque también para otras anomalías ${ }^{1}$. Su mecanismo de acción es el intercambio de los iones calcio o sodio (de acuerdo con el tipo de la resina) por el ion potasio una vez alcanzada la luz intestinal, de forma tal que este último aumenta la excreción por las heces².
Recibieron aprobación de la FDA para el tratamiento de la hiperpotasemia en 1958, después de que se publicara una serie de casos en la que se observó una reducción gradual de los valores de potasio con el uso de estas resinas ${ }^{3}$.

Aunque son poco frecuentes, los efectos adversos vinculados con este tratamiento pueden ser muy graves. Su uso se ha acompañado de necrosis intestinal y otras complicaciones gastrointestinales letales (ulceraciones, perforación, sangrado), que pueden sobrevenir desde pocas horas hasta días después del inicio del tratamiento con resinas de intercambio iónico, con dosis variables de 20 a $60 \mathrm{~g}$ al día ${ }^{4}$. Al principio se atribuyó este riesgo al uso concomitante de sorbitol, pero las revisiones sistemáticas, como la de Ziv Harel et al., demostraron que las soluciones sin sorbitol también se relacionaban con este tipo de complicaciones gastrointestinales ${ }^{5}$. Por lo regular, los factores de riesgo vinculados con el desarrollo de necrosis intestinal por resinas de intercambio iónico incluyen insuficiencia renal crónica, trasplantes y estados posoperatorios ${ }^{6}$. Este paciente tenía antecedente de enfermedad renal crónica y se encontraba en estado posoperatorio de drenaje de hematoma subdural, tal y como se describe en los factores de riesgo de las publicaciones médicas. La incidencia real de la necrosis intestinal por resinas de intercambio iónico no se ha dilucidado por completo; no obstante, Gertsman, et al. comunicaron una incidencia global de $0.27 \%$, que se eleva hasta $1.8 \%$ en pacientes posoperatorios ${ }^{7}$. El mecanismo de la necrosis inducida por estos fármacos aún no se conoce, pero podría deberse a un efecto directo de los propios cristales o al efecto osmótico añadido al sorbitol con el que se administran con frecuencia. Una posible explicación de su mayor incidencia en pacientes con insuficiencia renal señala que los valores de renina plasmática elevados predisponen a la isquemia mesentérica no oclusiva ocasionada por la vasoconstricción secundaria a la angiotensina ${ }^{8}$.

Los cambios fisiopatológicos observados en pacientes con perforación intestinal por resinas de intercambio iónico incluyen edema de la mucosa, formación de úlceras y seudomembranas y, en casos más graves, necrosis transmural. En la microscopia se reconocen cristales poligonales y basófilos con la tinción de hematoxilina-eosina y además se tiñen de color magenta con la coloración con ácido peryódico de Schiff ${ }^{6}$.

En Colombia no se han informado con anterioridad casos de necrosis intestinal secundaria al uso de resinas de intercambio iónico. En consecuencia, el caso presentado es de utilidad para sospechar este tipo de 
complicaciones en pacientes con cuadro de dolor abdominal o sangrado gastrointestinal y bajo tratamiento con resinas de intercambio iónico, sobre todo si poseen factores de riesgo adjuntos.

\section{Conclusión}

La necrosis intestinal relacionada con el tratamiento con resinas de intercambio iónico es infrecuente, pero potencialmente letal. La sospecha, la identificación de los factores de riesgo y el tratamiento oportuno multidisciplinario son cruciales para la sobrevivencia de los pacientes que desarrollan esta complicación.

\section{Agradecimientos}

Los autores agradecen al departamento de patología de la Clínica SOMA por su colaboración en el estudio histológico.

\section{Financiamiento}

El trabajo realizado fue autofinanciado.

\section{Conflicto de intereses}

Los autores declaran no tener ningún conflicto de intereses.

\section{Responsabilidades éticas}

Protección de personas y animales. Los autores declaran que para esta investigación no se han realizado experimentos en seres humanos ni en animales.
Confidencialidad de los datos. Los autores declaran que han seguido los protocolos de su centro de trabajo sobre la publicación de datos de pacientes.

Derecho a la privacidad y consentimiento informado. Los autores han obtenido el consentimiento informado de los pacientes y/o sujetos referidos en el artículo. Este documento obra en poder del autor de correspondencia.

\section{Bibliografía}

1. Sterns RH, Rojas M, Bernstein $P$, Chennupati S. lon-exchange resins for the treatment of hyperkalemia: are they safe and effective? JASN [Internet]. mayo de 2010 [citado 3 de agosto de 2020];21(5):733-5. Disponible en: http://www.jasn.org/lookup/doi/10.1681/ASN.2010010079

2. Dépret $F$, Peacock WF, Liu KD, Rafique $Z$, Rossignol $P$, Legrand $M$. Management of hyperkalemia in the acutely ill patient. Ann Intensive Care [Internet]. diciembre de 2019 [citado 3 de agosto de 2020];9(1):32. Disponible en: https://annalsofintensivecare.springeropen.com/articles/10.1186/s13613-019-0509-8

3. Evans BM, Jones NC, Milne MD, Yellowlees $\mathrm{H}$. lon-exchange resins in the treatment of anuria. Lancet. 1953;262:791-795.

4. Kelsey PB, Chen S, Lauwers GY. Case 37-2003: a 79-year-old man with coronary artery disease, peripheral vascular disease, end-stage renal disease, and abdominal pain and distention. Cabot RC, Harris NL, McNeely WF, Shepard J-AO, Ebeling SH, Ellender SM, et al., editores. $\mathrm{N}$ Engl J Med. [Internet]. 27 de noviembre de 2003 [citado 3 de agosto de 2020];349(22):2147-55. Disponible en: http://www.nejm.org/ doi/10.1056/NEJMcpc030031

5. Harel Z, Harel S, Shah PS, Wald R, Perl J, Bell CM. Gastrointestinal adverse events with sodium polystyrene sulfonate (kayexalate) use: a systematic review. The American Journal of Medicine [Internet]. marzo de 2013 [citado 14 de junio de 2020];126(3):264.e9-264.e24. Disponible en: https://linkinghub.elsevier.com/retrieve/pii/S0002934312008698

6. Rodríguez-Luna MR, Fernández-Rivera E, Guarneros-Zárate JE, Tueme-Izaguirre J, Hernández-Méndez JR. Cation exchange resins and colonic perforation. What surgeons need to know. International Journal of Surgery Case Reports. [Internet]. 2015 [citado 3 de agosto de 2020];16:102-5. Disponible en: https://linkinghub.elsevier.com/retrieve/pii/ S2210261215004204

7. Gerstman BB, Kirkman R, Platt R. Intestinal necrosis associated with postoperative orally administered sodium polystyrene sulfonate in sorbitol. Am J Kidney Dis. 1992;20:159-161.

8. Rogers FB, Li SC. Acute colonic necrosis associated with sodium polystyrene sulfonate (Kayexalate) enemas in critically ill patient: case report and review of the literature. J Trauma. 2001;51:395-397. 